\title{
Determination of Tetracycline Residues in Milk by High Performance Liquid Chromatography
}

\author{
Sneh Lata Chauhan, Priyanka, S.R. Garg and Vijay J. Jadhav* \\ Department of Veterinary Public Health \& Epidemiology, College of Veterinary Sciences \\ LUVAS, Hisar, Haryana- 125004, India \\ *Corresponding author
}

\section{A B S T R A C T}

\section{Keywords \\ Tetracycline residues, RP-HPLC, Milk, Solid phase extraction \\ Article Info \\ Accepted: \\ 20 January 2019 \\ Available Online: \\ 10 February 2019}

\begin{abstract}
A Reverse phase high-performance liquid chromatographic method (RP-HPLC) for the simultaneous determination of tetracycline, Oxytetracycline and chlortetracycline residues in milk has been developed. The determination of these antimicrobials was carried out using HPLC UV-VIS with a $\mathrm{C}_{8}$ hybrid column elution with a mobile phase composed of solvent A (water: formic acid as 1000:1 v/v) and solvent B (water: acetonitrile: formic acid as 100:900:1). Sample preparation involved protein precipitation followed by solid-phase extraction using a $\mathrm{C}_{18}$ cartridge. The method was validated and applied for the analysis of different type of milk samples commercialized in Hisar, Haryana. The limits of quantitation for all antimicrobials were below the maximum residue limit, which indicates that the method is appropriate for the determination of these antimicrobials in milk.
\end{abstract}

\section{Introduction}

Milk is an important constituent of human diet. It is consumed by all age groups particularly children and elderly people. India is the first ranker in milk production in the world with a total annual production of 146.3 million tonnes $(18.5 \%)$ and per capita availability $337 \mathrm{~g} /$ day (NDDB, 2016). The per capita availability of milk in Haryana is 835 g/day (DAHD, 2016). It is therefore important to provide due attention to the quality of milk produced and distributed to the consumers.

Antimicrobial agents mainly tetracycline's are widely used in food of animal origin for therapeutic purpose to treat diseases and control and used as a additive for the growth promotion and productive efficiency of the animal. The antimicrobial residues present in the food of animal origin above the established maximum residue limits (MRLs) indicates that good veterinary practices were not established and may lead to resistance to bacteria in humans. In milk, however, their presence may cause allergic reactions i.e. photosensitivity and risk of terratogenicity when administered during the first trimester of pregnancy in sensitive individuals and may interfere with starter cultures for cheese and other dairy products (Schenk and Callery, 
1998). Moreover, primary and permanent teeth discoloration often occurs when milk is consumed by infants. Tetracyclines, in particular chlortetracycline, have been routinely employed to prevent and treat mastitis in lactating dairy cows (JECFA, 2002). Chlortetracycline and Oxytetracycline are licensed as growth promoters for livestock in the United States (Meyer et al., 2000).

Throughout the world antimicrobial drug residues are known to be present in food but the milk is of greater concern to the human beings specially children that may lead to the resistance of microorganisms in gutflora (Ekuttan et al., 2007). Apart from the direct toxic effects on the consumers, antimicrobial residues cause propagation of antimicrobial drug resistant bacteria in the food chain, environment and the body system of humans as well as animals.

The rampant and indiscriminate use of antibiotics among the small-scale livestock keepers increases the possibility of transfer of antibiotic resistant bacteria from animals to humans and that may lead to various chronic diseases among the users of milk and milk products. Antimicrobial residues result in development of drug resistance in the gut flora in human beings.

Avoidance of antimicrobial residues in milk needs an important focus on the dairy industry. Lack of awareness of withdrawal times or increased use may lead to elevated levels of drug residues in the milk. The most common causes of occurrence of drug residues in milk are insufficient identification of treated cows, lack of knowledge about withdrawal periods and the failures of the hired staff.

The increasing use of antibiotic consumption in India is reflected by the emerging drug resistance problem while the regulations concerning the use of antibiotics in human and animals are still very poor. In addition to the health issue, the presence of antibiotic residues in milk may interfere with starter culture in the production of cheese and other fermented dairy products resulting in significant economic losses to the producers of milk and milk products (Katla et al., 2001).

The committee also recommended MRLs in milk of $100 \mu \mathrm{g} / \mathrm{l}$. The information on the occurrence of antibiotic drug residues in India is available only in the form of very limited academic research papers. Few such studies have demonstrated the presence of tetracycline residues in milk (Das et al., 2014; Gaurav et al., 2014; Kalla et al., 2015).

Different kinds of analytical methods are in practice to identify and quantify antibiotic residues in milk. While rapid screening methods (immunological or microbial inhibition assays) are commonly used to detect the presence of antimicrobials in food, more accurate chromatographic methods are required by the governmental regulatory agencies to identify and confirm the presence of these compounds. Such methods are always aimed at detection of individual analyte at a concentration lower than the specified MRL.

Conventional sample treatment protocols involve protein precipitation, centrifugation and analyte extraction, followed by clean-up of the extract over solid-phase cartridges. Many liquid chromatographic methods have been published for the determination of tetracyclines (Oka et al., 2000; Andersen et $a l ., 2005)$ but the work done in India is very limited. This paper focuses on the development and validation of a simple HPLC-UV method for the simultaneous determination of TC, OTC and CTC in milk which could be applied to quality control in the routine analysis. 


\section{Materials and Methods}

\section{Collection of samples}

A Total 100 milk samples were collected from Hisar and nearby areas. Among these, 40 raw milk samples were collected from local vendors and another 40 raw milk samples were collected from mini dairies (private milk collection centers). Twenty pasteurized milk samples of different brands were also collected from various retail shops. For each collected raw milk sample a quantity of about $100 \mathrm{~mL}$ was collected in a labeled sterilized bottle and stored at $-20^{\circ} \mathrm{C}$ till analysis.

\section{HPLC instrumentation and condition}

A Shimadzu prominence UFLC system equipped with DGU-20A5R degasser, SIL20A HT autosampler and LC-20AD pump connected to $\mathrm{C}_{8}$ column (Enable $4.6 \mathrm{~mm} \mathrm{x}$ $250 \mathrm{~mm}$ porosity $5 \mathrm{um}$ ) housed in CTO-10AS column oven with SPD-20A UV-VIS detector was used throughout the experiment. The system was controlled by Lab Solution Software.

\section{Chemicals and reagents}

The analytical standards of antimicrobials viz. tetracycline, Oxytetracycline and chlortetracycline, all having purity more than 98\%, were procured from Sigma-Aldrich. Supelclean ${ }^{\mathrm{TM}}$ LC-18 SPE Tube having bed wt. $500 \mathrm{mg}$ and volume $3 \mathrm{~mL}$ were also procured from Sigma-Aldrich. All HPLC grade solvents namely methanol, acetonitrile and iso-propyl alcohol (IPA) were procured from Fisher Scientific whereas anhydrous sodium sulphate was procured from Qualigens. HPLC grade water was prepared in the laboratory using Millipore (Bedford, MA, USA) Milli-Q system to give a resistivity of at least $18.2 \mathrm{M}$ $\Omega \mathrm{cm}$.

\section{Preparation of reagents}

\section{Mobile phase}

Mobile phase used for the instrumental analysis of tetracycline was composed of solvent A (water : formic acid as 1000:1 v/v) and solvent $\mathrm{B}$ (water : acetonitrile : formic acid as 100:900:1 v/v/v).

\section{Preparation of standards reagent solutions}

The primary standard solution of each antimicrobial was prepared by dissolving neat standards of TETs in methanol by using class A glassware (Final volume $25 \mathrm{ml}$ ) so that effective concentration remained more than $100 \mu \mathrm{g} / \mathrm{mL}$. Standard solutions of TETs were stored at $-18^{\circ} \mathrm{C}$. For preparation of individual secondary standard solutions, the maximum residue limits (MRLs) prescribed by European Union Commission (EU, 2010) and Codex Alimentarius Commission of WHO (Codex, 2015) for all antibiotics were considered. Based on these MRL values, a linearity range $(50,100,150,200,250 \mu \mathrm{g} / \mathrm{kg})$ was selected to cover the lowest MRLs for all the analyte molecules. Then, appropriate quantity of primary standard solution(s) was diluted to the required volume with same solvent to prepare individual secondary standard solution as well as standard mix.

\section{HPLC analysis}

HPLC-UV technique was standardized for detection of TETs viz. tetracycline, oxytetracycline and chlortetracycline from milk as per method described by Stolker et al., (2008) with slight modification. Mobile phase used for the instrumental analysis of tetracycline was composed of solvent A (water : formic acid as 1000:1 v/v) and solvent B (water : acetonitrile : formic acid as 100:900:1 v/v/v). The flow rate was $1 \mathrm{ml} / \mathrm{min}$. Detection of Tetracyclines was performed at UV detector at $280 \mathrm{~nm}$ wavelength. 


\section{Sample preparation}

The spiked milk samples for linearity as well as recovery studies were prepared by fortification of proportional quantities of ground blank milk samples were with standard mix at various concentrations viz. $50,100,150,200$ and $250 \mu \mathrm{g} / \mathrm{kg}$ and then subjected to extraction and cleanup procedure. Milk samples collected from market were processed as such.

\section{Results and Discussion}

In the present study, an analytical technique using high pressure liquid chromatography with UV (HPLC-UV) detector was standardized as per the sample processing method proposed by Stolker et al., (2008) with slight modifications. Standardized Tetracyclines compared with blank milk samples showed in chromatogram in Figure 1. Tetracyclines (TETs) such as tetracycline, oxytetracycline, chlortetracycline were detected by HPLC-UV technique. The standardized method was validated as per the ICH Hormonized Tripartite Guidelines ( ICH, 1998). This validated method was used for analysis of 100 randomly collected milk samples for the detection and quantitation of TETs.

\section{Standardization and validation studies}

i) System Precision: The system precision was evaluated by studying the reproducibility of the instrumental response with respect to retention time and area of an analyte. The percent relative standard deviation (RSD) for analyte was found to be in range of 0.64$1.12 \%$ for retention time of TETs. The percent relative standard deviation (RSD) for analyte was found to be in range of 3.0112.68 for area of TETs. The percent Relative Standard Deviation (\%RSD) for all analyte was found to be less than 0.07 percent for area and 0.02 percent for retention time. ii) Specificity: It was evaluated by visual observation of chromatograms of blank sample matrix and sample matrix spiked with standard mixture. For milk, chromatogramic signals at the retention times of TETs were absent in blank sample matrix.

iii) Linearity: The standard calibration curves of the analyzed TETs standards presented a good regression line ( $\mathrm{r} 2>0.99)$ in the range of explored concentrations i.e. from 50 to $250 \mu \mathrm{g} / \mathrm{kg}$. The graphs showing calibration curve of these standards, revealed that all concentrations of the TETs under study were collinear and thus calibration curves were further employed for the detection of analytes under study.

iv) Limit of detection (LOD) and limit of quantitation (LOQ): LOD and LOQ were determined on the basis of standard deviation of the blank. Measurement of the magnitude of the analytical background response was performed by the analysis of 10 blank samples and calculating the standard deviations of these responses. Table 1 summaries the LOD and LOQ obtained for each analytes of TETs group. Perusal of tables clearly indicates that the LOD and LOQ for individual analytes were well below their respective MRLs indicating that the method was able to detect the given antibiotics at sufficiently low level.

v) Accuracy: The accuracy in terms of percent recovery of each analytes of TETs group at five different fortification levels $(50,100$, 150,200 and $250 \mu \mathrm{g} / \mathrm{kg}$ ) was evaluated for milk and the results are presented in Table 2. Satisfactory results were found in almost all instances. Recoveries for all analyte-matrix combinations ranged between $71-110 \%$ in milk. However, in general, the antibiotics gave acceptable recoveries within the mentioned validation interval as per legislation (EU, 2002) between 70 and 110 percent. 
vi) Precision: The precision was assessed, at five concentration levels $(50,100,150,200$ and $250 \mu \mathrm{g} / \mathrm{kg}$ ) by the recovery studies. Repeatability and intermediate precision values, expressed as relative standard deviation (CV percent) were found less than 10.51 for all analytes of TETs (Table 3 ).

Overall the multiresidue method followed for multiresidue detection and quantification of TETs antibiotic residues in milk was subjected to rigorous validation parameters. The system precision values indicated a good consistency in response by the HPLC instrument used during present study. A good linearity was noted for standards and spiked milk samples. Absence of interfering peaks in blank samples indicates good specificity of extraction and clean up method. Accuracy and precision of the method were in accepted range in comparison with international guidelines. These results of validation studies clearly demonstrated that the present method is suited for routine analysis of TETs in milk.

\section{Determination of residues of TETs in milk}

After successful standardization and validation of techniques for detection of TETs residues the extraction, detection and quantification were carried out on 100 samples of milk collected from Hisar city. The overall occurrence of TETs residues is presented in Table 4.

Table.1 Limit of detection (LOD) and limit of quantification (LOQ) for TETs

\begin{tabular}{|l|l|l|l|}
\hline Group of antimicrobials & Analytes & LOD $(\boldsymbol{\mu g} / \mathbf{k g})$ & $\mathbf{L O Q}(\boldsymbol{\mu g} / \mathbf{k g})$ \\
\hline Tetracyclines & Oxytetracycline & 48 & 98 \\
\hline & Tetracycline & 44 & 94 \\
\hline & Chlortetracycline & 8 & 23 \\
\hline
\end{tabular}

Table.2 Accuracy of method for detection of TETs residues in milk

\begin{tabular}{|c|c|c|c|c|c|}
\hline \multirow[t]{2}{*}{ Analytes } & \multicolumn{2}{|c|}{$\begin{array}{l}\text { Accuracy (\% Averag } \\
\text { Concentrations }(\mu \mathrm{g} / \mathrm{kg})\end{array}$} & \multicolumn{3}{|c|}{ Recovery \pm SD) at various spiked } \\
\hline & 50 & 100 & 150 & 200 & 250 \\
\hline Oxytetracycline & $109.6 \pm 4.6$ & $110.8 \pm 8.48$ & $107 \pm 11.79$ & $105 \pm 3.84$ & $109.6 \pm 9.67$ \\
\hline Tetracycline & $108 \pm 3.68$ & $101 \pm 7.02$ & $98 \pm 10.36$ & $101 \pm 5.52$ & $87.1 \pm 7.41$ \\
\hline Chlortetracycline & $65.5 \pm 5.40$ & $73.5 \pm 6.18$ & $71.18 \pm 7.27$ & $61 \pm 1.81$ & $64.6 \pm 6.14$ \\
\hline
\end{tabular}

Table.3 Precision of method for detection of TETs residues in milk

\begin{tabular}{|l|l|l|l|l|l|}
\hline \multirow{2}{*}{ Analytes } & \multicolumn{4}{|l|}{$\begin{array}{l}\text { Precision } \\
\text { spiked Concentrations }(\boldsymbol{\mu g} / \mathbf{k g})\end{array}$} \\
\cline { 2 - 6 } & $\mathbf{5 0}$ & $\mathbf{1 0 0}$ & $\mathbf{1 5 0}$ & $\mathbf{2 0 0}$ & $\mathbf{2 5 0}$ \\
\hline Oxytetracycline & 4.26 & 7.65 & 9.7 & 3.66 & 8.81 \\
\hline Tetracycline & 3.41 & 6.93 & 9.49 & 5.51 & 8.48 \\
\hline Chlortetracycline & 8.24 & 8.41 & 9.8 & 2.99 & 9.46 \\
\hline
\end{tabular}


Table.4 Mean concentration of TETs residues in milk

\begin{tabular}{|l|l|l|l|l|l|}
\hline \multirow{2}{*}{$\begin{array}{l}\text { Group of } \\
\text { antimicrobials }\end{array}$} & Analytes & \multicolumn{4}{|l|}{ Mean concentration $(\boldsymbol{\mu g} / \mathbf{K g})$} \\
\cline { 3 - 6 } & $\begin{array}{l}\text { Raw milk- } \\
\text { Vendor } \\
(\mathbf{n = 4 0})\end{array}$ & $\begin{array}{l}\text { Raw milk- } \\
\text { Dairy } \\
(\mathbf{n = 4 0})\end{array}$ & $\begin{array}{l}\text { Pasteurized } \\
\text { milk } \\
(\mathbf{n = 2 0})\end{array}$ & $\begin{array}{l}\text { Total } \\
(\mathbf{n}=\mathbf{1 0 0})\end{array}$ \\
\hline \multirow{2}{*}{ Tetracyclines } & Oxytetracycline & 25.49 & - & 11.26 & 12.45 \\
\cline { 2 - 6 } & Tetracycline & 2.78 & - & - & 1.11 \\
\hline & Chlortetracycline & 16.68 & 2.55 & - & 7.69 \\
\hline
\end{tabular}

Table.5 Comparison of TETs residue levels in milk samples with the national and International MRLs

\begin{tabular}{|l|l|l|l|l|}
\hline \multirow{2}{*}{ Analyte } & \multicolumn{2}{|l|}{ International MRLs } & \multicolumn{2}{l|}{$\begin{array}{l}\text { Number of samples } \\
\text { violating MRLs }\end{array}$} \\
\cline { 2 - 4 } & EU (2010) $(\boldsymbol{\mu g} / \mathbf{k g})$ & Codex $(\mathbf{2 0 1 5})(\boldsymbol{\mu g} / \mathbf{l})$ & EU & Codex \\
\hline Oxytetracycline & 100 & $100^{*}$ & 5 & 5 \\
\hline Tetracycline & 100 & & 0 & 0 \\
\hline Chlortetracycline & 100 & & 0 & 0 \\
\hline
\end{tabular}

NE $=$ Not established

*This tolerance includes both the sum and the individual residues of chlortetracycline, oxytetracycline and tetracycline. The sum of the tetracyclines present should not exceed $100 \mu \mathrm{g} / \mathrm{l}$ (Codex, 2015)

Fig.1 Chromatogram of solvent blank and standard mix of tetracyclines

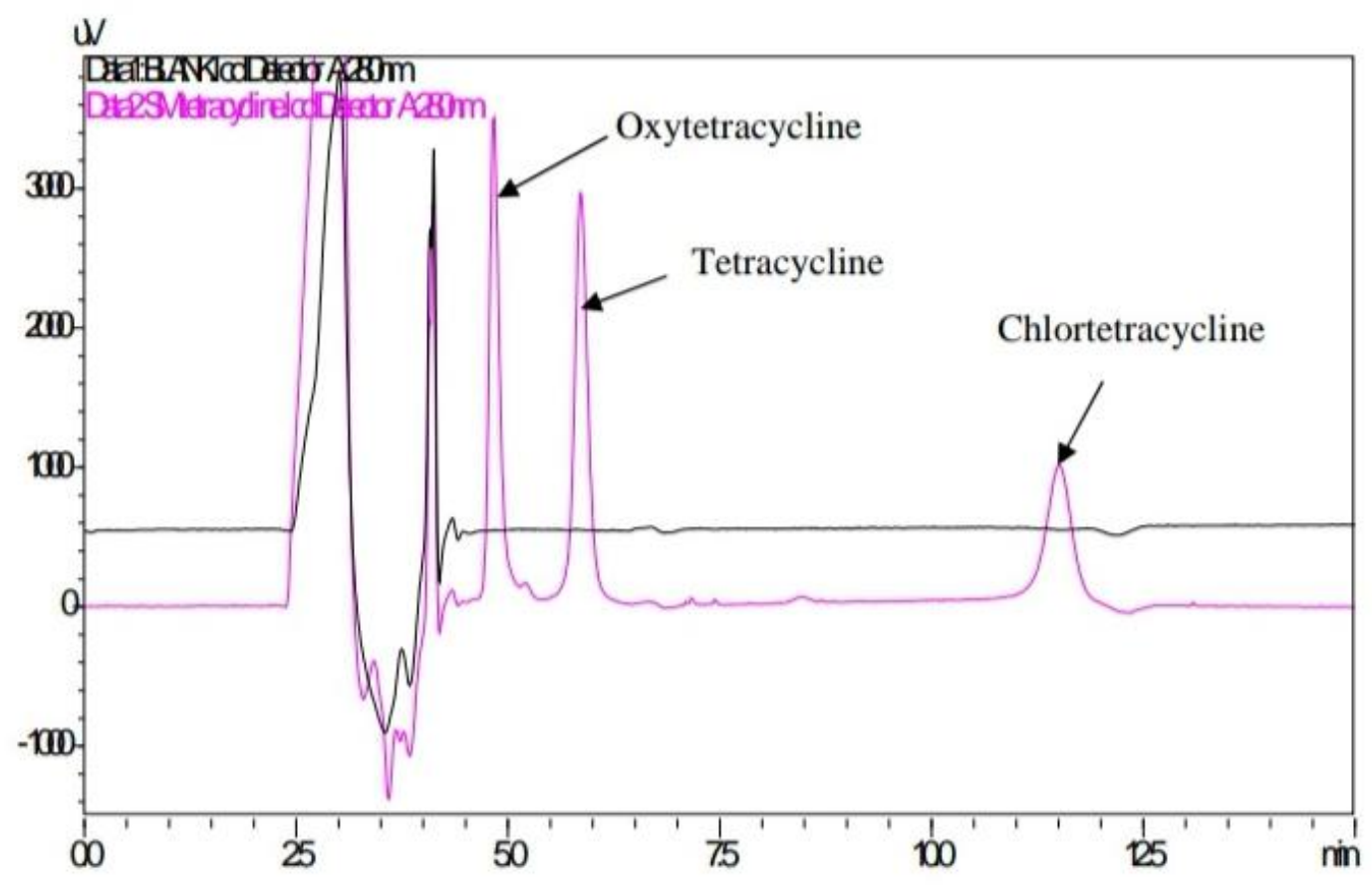


In the present study, maximum numbers of milk samples were found to be contaminated with CTC (9\%) residues followed by oxytetracycline $(6 \%)$ and tetracycline $(3 \%)$. Raw milk samples from vendors showed presence of all three TCs while the milk samples from mini dairies showed presence of all except oxytetracycline residues. Pasteurized milk samples showed presence of only oxytetracycline. This trend shows the effect of mixing and dilution of antimicrobial residue contaminated milk with uncontaminated milk.

Mean concentration for each analyte is provided in Table 4 in milk samples. The results revealed that absolute mean concentration of tetracyclines was found to be $21.25 \mu \mathrm{g} / \mathrm{kg}$ which was mostly contributed by oxytetracycline $(12.45 \mu \mathrm{g} / \mathrm{kg})$ followed by chlortetracycline $(7.69 \mu \mathrm{g} / \mathrm{kg})$ and tetracycline $(1.11 \mu \mathrm{g} / \mathrm{kg})$. Alomirah et al., (2007) reported that $29.1 \%$ of the analyzed fresh milk samples were above the MRL for tested residues, with tetracycline as the dominant residue. Similarly, in the study conducted by Bilandzic et al., (2011), the highest tetracycline level detected was $49.5 \mu \mathrm{g} / \mathrm{kg}$. However, the mean tetracycline concentration $(2.83 \mu \mathrm{g} / \mathrm{kg})$ was more than 35 times lower than the MRL level. Whereas, in the present study, the mean concentration of tetracycline residues was found to be less than the values obtained in the above study.

On the contrary to the findings of present study, TCs were detected with much higher frequency in other states of India. In general, out of all samples, 5\% samples were found to be exceeding the MRL with respect to tetracyclines (Table 5). Sudershan and bhat (1995) found OTC residues in 9\% of market milk samples and $73 \%$ in individual animal samples in Hyderabad. Gaurav et al., (2014) studied the occurrence of tetracycline residues in milk samples collected from various part of Punjab by ELISA method and found the residues in $13 \%$ samples. Kalla and coworkers (2015) in Andhra Pradesh studied the prevalence of antibiotic residues in raw milk and found $51 \%$ milk samples positive for TETs. However, Nirala et al., (2017) found tetracycline residues in only $3.3 \%$ milk samples from various districts of Bihar which is comparatively in lesser proportion than the present study.

Occurrence of tetracycline residues has also been reported from various parts of the world. In Brazil, Bando et al., (2009) and Zanella et al., (2010) reported significantly high presence of TC antibiotic with $41(27.2 \%)$ and 48 samples $(18.5 \%)$ contaminated with TC residues. Ahmed et al., (2015) found tetracycline residues in Egypt in 30\% milk sample with the mean concentration of 23.62 $\pm 7.01 \mu \mathrm{g} / \mathrm{L}$. Syit (2011) reported OTC residues in Ethiopia in $70.58 \%$ milk samples above MRL and the mean residue level of OTC was $142.00 \mu \mathrm{g} / \mathrm{L}$. TC residues were detected in the milk from various parts of the world with much higher frequency than the present study (Navratilova et al., 2009; Abbasi et al., 2011 and Elizabeta et al., 2011).

Rasooli et al., (2014) examined the presence of tetracycline residues in 432 pasteurized milk samples in Iran and found 7 samples above the MRL. All the samples above tolerance limits were recorded in association with vendor milk and some of the dairy or pasteurized milk sample was found to have antimicrobial concentration above MRLs. This might be due to the dilution effect of bulking on residue concentration in dairy and pasteurized milk samples i.e. mixing of positive milk with negative milk at a larger level in dairy plants.

\section{Acknowledgements}

Authors acknowledge the help provided by Dr. Pallavi and Dr. Himani. 


\section{References}

Abbasi, M.M., Babaei, H., \&Ansarin, M. 2011. Simultaneous determination of tetracyclines residues in bovine milk samples by solid phase extraction and HPLC-FL method. Advanced Pharmaceutical Bulletin. 1:34.

Ahmed, K.M., Hafez, R.S., Morgan, S.D. and Awad, A.A. 2015. Detection of some chemical hazards in milk and some dairy products. Afr. J. Food Sci. 9:187193.

Alomirah, H., Al-Mazeedi, H., Al-Zenki, S., Al-Aati, T., Al-Otaibi, J., Al-Batel, M. and Sidhu, J. 2007. Prevalence of antimicrobial residues in milk and dairy products in the state of Kuwait. Journal of Food Quality. 30:745-763.

Anderson, W., Roybal, J., Gonzales, S., Turnipseed, S., Pfenning, A., and Kuck, L. 2005. Determination of tetracycline residues in shrimp and whole milk using liquid chromatography with ultraviolet detection and residue confirmation by mass spectrometry. Analytica Chimica Acta. 529:145-150.

Bilandzic, N., Solomun B, Varenina K.I. and Jurković, Z. 2011. Concentrations of veterinary drug residues in milk from individual farms in Croatia. Mljekarstvo / Dairy. 61:260-267.

CODEX., 2015. Codex Alimentarius Commission. Maximum residue limits for veterinary drugs in foods. Available at http, //www.codexalimentarius.net/ vetdrugs/data/index.html

Das, S., Kumar, N., Vishweswaraiah, R.H., Haldar, L., Gaare, M., Singh, V.K. and Puniya, A.K. 2014. Microbial based assay for specific detection of $\beta$-lactam group of antibiotics in milk. Journal of Food Science and Technology. 51:1161-1166.

DAHD., 2016. Statistical information related to animal husbandry sector. Department of Animal Husbandry and Dairying, Haryana. 2016. Available at http, //www.pashudhanharyana.gov.in/html/s tatistics.html

Ekuttan, C. E., Kang'ethe, E. K., Kimani, V. N., and Randolph, T. F. 2007. Investigation on the prevalence of antimicrobial residues in milk obtained from urban smallholder dairy and nondairy farming households in Dagoretti Division, Nairobi, Kenya. East African medical journal, 84(11 Suppl), S87-91.

Elizabeta, D.S., Zehra, H.M., Biljana, S.D., Pavle, S. and Risto, U. 2011. Screening of veterinary drug residues in milk from individual farms in Macedonia. Macedonian Veterinary Review. 34(1): 5-13.

EU. European Union Council Regulation No. 37/2010 of 22 December 2009. 2010. On pharmacologically active substances and their classification regarding maximum residue limits in foodstuffs of animal origin. Official Journal of the European Communities L15, 1-72.

Gaurav, A., Gill, J.P.S., Aulakh, R.S. and Bedi, J.S. 2014. ELISA based monitoring and analysis of tetracycline residues in cattle milk in various districts of Punjab. Veterinary World. 7:26-29.

Kalla, A., Kutcharlapati, S.R., Gannavarapu, S.B. and Layam, A. 2015. Isolation and Identification of Specific Pathogens, Presence of Antibiotics, Aflatoxins, Pesticide Residues and Industrial Contaminants in Supply Chain of Milk in Selected Coastal Districts of Andhra Pradesh. Advances in Bioscience and Biotechnology. 6:330-344.

Katla, A. K., Kruse, H., Johnsen, G., and Herikstad, H. 2001. Antimicrobial susceptibility of starter culture bacteria used in Norwegian dairy products. International journal of food microbiology. 67(1-2):147-152. 
Meyer, M.T., Bumgarner, J.E., Varns, J.L., Daughtridge, J.V., Thurman, E.M., Hostetler, K.A. 2000. Use of radioimmunoassay as a screen for antibiotics in confined animal feeding operations and confirmation by liquid chromatography/mass spectrometry. Sci. Total Environ. 248:181-187.

Navratilova, P., Borkovcova, I., Dračkova, M., Janštova, B. and Vorlova, L. 2009. Occurrence of tetracycline, chlortetracyclin, and oxytetracycline residues in raw cow's milk. Czech Journal of Food Sciences. 27:379-385.

NDDB., 2016. Milk production in India. National Dairy Development Board. Available at http, //www.nddb.org/information/stats/milk prodindia.

Nirala, R.K., Anjana, K., Mandal, K.G. and Jayachandran, C. 2017. Persistence of Antibiotic Residue in Milk under Region of Bihar, India. International Journal of Current Microbiology and Applied Sciences. 6: 2296-2299.

Oka, H., Ito, Y., and Matsumoto, H. 2000. Chromatographic analysis of tetracycline antibiotics in foods. Journal of Chromatography A. 882(1-2): 109133.

Schenk, F. J., and Callery, P. S. 1998. Chromatographic methods of analysis of antibiotics in milk. Journal of Chromatography A. 812: 99-109.

Syit, D.A. 2011. Detection and determination of oxytetracycline and penicillin G antibiotic residue levels in bovine bulk milk from debrezeit and nazareth dairy farms. In, Proceedings of the $1^{\text {st }}$ International Technology, Education and Environment Conference, Omoku, Nigeria.

Stolker, A.A., Rutgers, P., Oosterink, E., Lasaroms, J.J., Peters, R.J., Van Rhijn, J.A. and Nielen, M.W. 2008. Comprehensive screening and quantification of veterinary drugs in milk using UPLC-ToF-MS. Analytical and Bioanalytical Chemistry. 391:23092322.

Sudershan, R.V. and Bhat, R.V. 1995. A survey on veterinary drug use and residues in milk in Hyderabad. Food Additives and Contaminants. 12: 645650.

Van Boeckel, T.P., Brower, C., Gilbert, M., Grenfell, B.T., Levin, S.A., Robinson, T.P., and Laxminarayan, R. 2015. Global trends in antimicrobial use in food animals. Proceedings of the National Academy of Sciences of the United States of America. 112: 56495654.

Zanella, G.N., Mikcha, J.M.G., Bando, E., Siqueira, V.L.D., and MachinskiJr, M. 2010. Occurrence and antibiotic resistance of coliform bacteria and antimicrobial residues in pasteurized cow's milk from Brazil. Journal of Food Protection. 73: 1684-1687.

\section{How to cite this article:}

Sneh Lata Chauhan, Priyanka S.R. Garg and Vijay J. Jadhav. 2019. Determination of Tetracycline Residues in Milk by High Performance Liquid Chromatography. Int.J.Curr.Microbiol.App.Sci. 8(02): 2763-2771. doi: https://doi.org/10.20546/ijcmas.2019.802.324 